\title{
Nanoparticle (NP) Delivery of Chemotherapy Drugs to Prostate Cancer Patients
}

\author{
Toluleke Oloruntobi Famuyiwa ${ }^{1 *}$ and James Kwasi Kumi-Diaka \\ Department of Biological Sciences, College of Science, Florida Atlantic University, USA \\ *Corresponding author: Toluleke Oloruntobi Famuyiwa, Department of Biological Sciences, College of Science, Florida Atlantic University, Florida, USA
}

Submission: 酒 August 08, 2018; Published: 海 September 07, 2018

\begin{abstract}
The use of nanoparticles (NPs) for enhanced drug delivery in prostate cancer treatment has been extensively explored in many studies. However, there are serious limitations to nanoparticle delivery of drugs to prostate cancer. It has become increasingly obvious that the physicochemical properties of the nanoparticles dictate the volume of the nanoparticles needed for the drug delivery. The volume of nanoparticle used in turn determines therapeutic efficacy of the drug and the nanoparticle delivery system. In this review, we reflect on the physical chemistry of NP-mediated drug delivery to the target cell/tissue during prostate cancer treatment.
\end{abstract}

Keywords: Prostate cancer; Patients; Nanoparticles; Delivery system; Therapeutic efficiency; Drug; NP-Mediated drug; Biodegradable copolymers; Physicochemical properties; Moieties; Oligonucleotides; P-glycoprotein; Bio-compatibilities; 3-Bromopyruvate; SC-514; Quercetin; Prostate specific antigen (PSMA)

Abbreviations: NP: Nanoparticles; 3-BPA: 3-Bromopuruvate; PLGA: Poly lactic-co-glycolic Acid; FDA: Food and Drug Administration; EMA: European Medicine Agency; OVA: Ovalbumin; TLR4: Toll like Receptor-4; TAA: Tumor Associated Antigen; RNA: Ribonucleic Acid; CPs: Control Nanoparticles; PSMA: Prostate-Specific Membrane Antigen

\section{Review}

Controlled drug delivery systems have attracted diverse research interests over the years [1-5]. This system is a useful mechanism in prostate cancer treatment. Designing nanoparticles that selectively recognize and kill prostate cancer cells in the body, remains an innovative concept [6-8]. The knowledge has motivated researchers to build nanoparticles with unique physicochemical properties such as, size, shape and surface chemistry. These nanoparticles are programmed with a multitude of biological and medical functions $[9,10]$ that are vital for effective treatment of prostate cancer.

The prospects of using nanoparticle (NP) in clinical oncology are expanding every day. Numerous nanoparticles have been developed to increase bioavailability profiles of hydrophobic chemotherapeutic drugs such as SC-514 and Quercetin. This mode of delivery enables the administration of lower doses of the drug (SC-514 and Quercetin) and thereby minimizing the adverse effects found with systemic drug administration in clinical practices. Nanomedicine has increased the quality of life of prostate cancer patients [11]. Nanoparticle study is expected to lead to major advances involving the functionalizing the surface of the nanoparticles to improve the sensitivity and specificity of existing anti-cancer drugs. Hence, nanoparticle drug delivery studies are promising platforms for the synthesis of cell-specific anticancer agents [12].

Nanoparticles of biodegradable copolymers are emerging as a promising drug delivery vehicle for prostate cancer treatment. These nano-carrier systems possess diverse range of beneficial features including the significant reduction in volume of drug. These particles also have the ability to develop better pharmacokinetics [13]. Nanoparticles are therapeutic enhancers of anticancer drugs through passive or active targeting while reducing the lethal effects of drugs to healthy cells and tissues [14].

The use of NP for prostate cancer treatment has made a revolutionary impact in the area of therapeutics [15]. These release systems have been shown to enhance the stability of various therapeutic agents such as tiny hydrophobic moieties, peptides, and oligonucleotides [16]. Due to the small size of nanoparticles, the surface area to volume ratio is very large and can lead to the release of drugs at concentrations high enough to kill prostate and other cancer cells [17]. The drug release mechanisms are mainly effected by $\mathrm{pH}$, temperature, light, and hydrophobicity, among other factors [18]. The non-covalently bonded drugs have been released through hydrophobicity-induced phenomena via applying hydrophobic/ hydrophilic forces to the nanocarriers $[19,20]$. The high surface 
area of the nanoparticles allows enhanced drug loading efficiency and targeted drug delivery with minimum leakage and toxicity [21].

Nanoparticles present prolonged distribution of bioactive particles with higher body retention and permeability [22]. These are different types of nanoparticles, including organic nanoparticles (polymers, dendrimers, solid lipid), inorganic nanoparticles (magnetic iron oxide, quantum dots of various mineral compositions of silica), and protein-based nanoparticles (viruses and albumin) [18]. Each type could allow distribution of bioactive molecules to the target organs of interest [22]. In this article, the focus is on polymeric nanoparticles.

Polymeric nanoparticles (NP) show some advantages with respect to other drug delivery systems; such as more stability during storage [23]. Polymeric nanoparticles could reduce the multi-drug resistance that characterizes many anti-cancer drugs, by a mechanism of internalization of the drug [24] thereby reducing efflux from cells mediated by the P-glycoprotein [25]. A study showed that Docetaxel (DTX) and curcumin (CUR) loaded lipid-polymer hybrid nanoparticles (LPNs) impacted the highest cytotoxicity and synergistic effect of drug treatments in tumor cells in vitro [26]. Also, the conjugation of targeting peptide to superparamagnetic iron oxide nanoparticles (SPIONs), results in more precise delivery of these agents to tumor sites [27].

It has been reported that gold NP attached to 3-Bromopyruvate (3-BPA) could target the mitochondrial membrane potential more selectively and precisely than treatment with 3-BPA alone $[28,29]$. Interestingly, gold NP preferentially kill cancer cells more than normal mesenchymal stem cells due to higher mitochondrial membrane potential in cancer cells compared to normal cells $[28,29]$. Furthermore, oral administration of surface modified Poly (lactic-co-glycolic acid) (PLGA) NP containing capecitabine delivers the drug to the prostate effectively and precisely than conventional treatment approach [30]. This drug formulation significantly improves patient condition by reducing dosing frequency of conventional treatment modality. This delivery method helps in better management of prostate cancer [30]. Additionally, PLGA adjuvant NP systems are reported to elicit a strong $\mathrm{T}$ cell immune response, using 100 -fold lower doses $(0.05 \mu \mathrm{g})$ of $\mathrm{CpG}$ oligodeoxynucleotide antigen. In previous studies, PLGA NP systems showed significantly higher cytokine secretion (up to 10fold), as well as a comparative antibody response to abnormal body physiology [31]. Inevitably, PLGA systems may be further developed for tailored drug delivery in both chemo- and immunotherapy in prostate cancer treatment regimen.

PLGA is a biocompatible member of the aliphatic polyester polymer family of biodegradable polymers. It has long been a popular choice for drug delivery applications since its approval by the Food and Drug Administration (FDA) for use in humans [32]. PLGA nanoparticle can transport hydrophobic drugs such as SC514 in an aqueous environment for delivery within minutes. The drug enters the cell just by "slight contact" with the phospholipid layer of the cell [33]. Biodegradable NP constructed from PLGA polymers are widely used as antigen carriers/adjuvants due to its biocompatible and biodegradable characteristics. Safety profile and use has been approved by both the US FDA and the European Medicine Agency (EMA) [34]. PLGA offers unique advantages and properties for drug delivery purposes, like the world-wide approval for medical use, biodegradability, biocompatibility, and controlled release [30]. However, there are considerable challenges that must be overcome in developing PLGA-based NP systems for drug delivery application. The challenges: targeting the diseased tissue, cellular uptake together with pre-programmed intracellular trafficking, and escaping the reticuloendothelial system are not manageable by a single polymer [30]. The contact of PLGA nanoparticle with the body and the impact are mediated via the surface of the PLGA nanoparticles. PLGA-based NPs have poor loading capacity and display sudden release of drugs to unwanted tissues and/or cells.

Surface modification of PLGA-particles by grafting with selected biomimetic ligands can meet some of these challenges. Surface modification can lead to a more efficacious medication. Effective medications can reduced side effects and improve patient's treatment outcome [30]. Surface modification of PLGA nanoparticles can deliver the drug in a controlled manner throughout the prostate by using a much reduced dosing schedule to increase the therapeutic efficiency [30]. Several modifications have been utilized to overcome these problems. A pH sensitive PLGA NP system was developed for rapid release of ovalbumin (OVA) antigen in acidic environments to improve immune response [35]. The pH-based drug release has been used for tumor targeting drug release. This release mechanism is effective because tumor tissues possess lower $\mathrm{pH}$ than normal tissues [36-38].

Another modification to improve the drug release is the biomolecular supported process [39]. This method is commonly grouped into three parts: ligand exchange mediated release [40], enzymatic release [41,42], and chemical reduction-based release [43]. Delivery of dual or triple antigens in PLGA-NPs, co-delivery of Toll like Receptor-4 (TLR 4) ligand and Tumor Associated Antigen (TAA) using PLGA-based NPs [44], may occur through ligand exchange mediated release mechanism. Hydrophilic or hydrophobic interactions by heating, may occur through chemical reduction mechanism. This latter interaction permits encapsulated drugs to diffuse out of the matrix and into the target cell or organ [18].

The major considerations in determining the method for drug delivery are the parameters that influence bio-compatibilities and bioactivities of nanoparticles. Particle size is one of the most important parameters that determine bio-compatibilities and bioactivities of nanoparticles. Particle size has a direct relevance to the stability of drug formulations [30]. The delivery efficiency of PLGA nanoparticle is impacted by all the drug releasing factors mentioned above. 
A study showed that Epigallocatechin 3-gallate (EGCG) loaded NP system functionalized with a Prostate -specific membrane antigen (PSMA) inhibitor on the surface significantly enhances binding to PSMA with respect to the nonfunctionalized NP. This led to an increased antiproliferative activity in in vitro assays toward PSMA-positive PCa cells, without affecting normal cell viability [45]. Recommendations to augment delivery efficiency of PLGA nanoparticles to prostate cancer include; surface functionalization of PLGA NPs with the A10 2'-fluoropyrimidine ribonucleic acid (RNA) aptamers (that recognize the PSMA on prostate cancer cells) and biotin to modify the surface of PLGA nanoparticle [46]. Biotin is a small molecule that can induce efficient receptor-mediated endocytosis [47]. Growing evidence suggests a role for biotin in cell signaling, gene expression, and chromatin remodeling, together with its potential involvement in inhibiting prostate cancer cell proliferation [48]. Previous work indicated that molecular modification of PLGA nanoparticles with biotin did not change the process of nanoparticle nucleation and growth in solution. There was no evidence of change in shape, size or aggregation of biotinylated nanoparticle compared to the control nanoparticles (CPs) [49]. This observation further justifies the suggested use of biotin to functionalize PLGA nanoparticle.

In conclusion, clinical effectiveness of nanoparticles has many limitations [50]. A central strategy for addressing all these issues is to increase the delivery efficiency and specificity of the drug on the targeted tumor. If delivery efficiencies increase from $1 \%$ to $10 \%$, the volume of nanoparticles needed to release the same concentration of drug decrease from $90 \mathrm{ml}$ to $9 \mathrm{ml}$ [50]. The lower the volume of nanoparticles used in prostate cancer treatment the lesser the chances that healthy tissues and cells will be impacted with toxicities. The reduction in the volume of nanoparticles in contact with healthy cells and tissues implies increase therapeutic efficiency of the drug and the nanoparticle delivery system.

\section{References}

1. Pakulska MM, Miersch S, Shoichet MS (2016) Designer protein delivery: from natural to engineered affinity-controlled release systems. Science 351(6279): aac4750

2. Lu Y, Aimetti A, Langer R, Gu Z (2016) Bioresponsive materials. Nat Rev Mater doi:10.1038/natrevmats.2016.75.

3. Raavé R, van Kuppevelt TH, Daamen WF (2018) Chemotherapeutic drug delivery by tumoral extracellular matrix targeting. J Control Release 274: 1-8.

4. Meléndez-Ortiz I, Varca GHC, Lugão AB, Bucio E (2015) Smart polymers and coatings obtained by ionizing radiation: synthesis and biomedical applications. Open J Polym Chem 5(3): 17-33.

5. Koshy ST, Zhang DKY, Grolman JM, Stafford AG, Mooney DJ (2018) Injectable nanocomposite cryogels for versatile protein drug delivery. Acta Biomater 65: 36-43.

6. Peer D, Karp JM, Hong S, Farokhzad OC, Margalit R, et al. (2007) Nanocarriers as an emerging platform for cancer therapy. Nat Nanotechnol 2(12): 751-760.
7. Rao W, Wang H, Han J, Zhao S, Dumbleton J, et al. (2015) Chitosandecorated doxorubicin-encapsulated nanoparticle targets and eliminates tumor reinitiating cancer stem-like cells. ACS Nano 9(6): 5725-5740.

8. Min Y, Caster JM, Eblan MJ, Wang AZ (2015) Clinical translation of nanomedicine. Chem Rev 115(19): 11147-11190.

9. Nel AE, Mädler L, Velegol D, Xia T, Hoek EM, et al. (2009) Understanding biophysicochemical interactions at the nano-bio interface. Nat Mater 8(7): 543-557.

10. Albanese A, Tang PS, Chan WC (2012) The effect of nanoparticle size, shape, and surface chemistry on biological systems. Annu Rev Biomed Eng 14: 1-16.

11. Vizirianakis IS (2011) Nanomedicine and personalized medicine toward the application of pharmacotyping in clinical practice to improve drugdelivery outcomes. Nanomedicine 7(1): 11-17.

12. Boulaiz H, Alvarez PJ, Ramirez A, Marchal JA, Prados J, et al. (2011) Nanomedicine: application areas and development prospects. Int J Mol Sci 12(5): 3303-3321.

13.Zhang H, Chen T, Jiang J, Wong YS, Yang F, et al. (2011) Seleniumcontaining allophycocyanin purified from selenium-enriched Spirulina platensis attenuates AAPH-induced oxidative stress in human erythrocytes through inhibition of ROS generation. J Agric Food Chem 59(16): 8683-8690.

14. Chen T, Wong YS (2008) In vitro antioxidant and antiproliferative activities of selenium-containing phycocyanin from selenium-enriched Spirulina platensis. J Agric Food Chem 56(12): 4352-4358.

15. Ahmed EA, Omar HM, elghaffar SKh, Ragb SM, Nasser AY (2011) The antioxidant activity of Vitamin C, DPPD and l-cysteine against Cisplatininduced testicular oxidative damage in rats. Food Chem Toxicol 49(5): 1115-1121.

16. Caffrey PB, Frenkel GD (2000) Selenium compounds prevent the induction of drug resistance by cisplatin in human ovarian tumor xenografts in vivo. Cancer Chemother Pharmacol 46(1): 74-78.

17. Li S, Zhou Y, Wang R, Zhang H, Dong Y, et al. (2007) Selenium sensitizes MCF-7 breast cancer cells to doxorubicin-induced apoptosis through modulation of phospho-Akt and its downstream substrates. Mol Cancer Ther 6(3): 1031-1038.

18. Chaudhary S, Umar A, Mehta SK (2014) Surface functionalized selenium nanoparticles for biomedical applications. J Biomed Nanotechnol 10(10): 3004-3042.

19. Kumi-Diaka J, Merchant K, Haces A, Hormann V, Johnson M (2010) Genistein-selenium combination induces growth arrest in prostate cancer cells. J Med Food 13(4): 842-850.

20. Gang J, Park SB, Hyung W, Choi EH, Wen J, et al. (2007) Magnetic poly Î $\mu$ caprolactone nanoparticles containing $\mathrm{Fe} 3 \mathrm{O} 4$ and gemcitabine enhance anti-tumor effect in pancreatic cancer xenograft mouse model. J Drug Target 15(6): 445-453.

21. Jayaprakash V, Marshall JR (201) Selenium and other antioxidants for chemoprevention of gastrointestinal cancers. Best Pract Res Clin Gastroenterol 25(4-5): 507-518.

22. Pu W, Wang D, Zhou D (2015) Structural characterization and evaluation of the antioxidant activity of phenolic compounds from astragalus taipaishanensis and their structure-activity relationship. Sci Rep 5: 13914.

23. Müller RH, Jacobs C, Kayser O (2001) Nanosuspensions as particulate drug formulations in therapy: Rationale for development and what we can expect for the future. Adv Drug Deliv Rev 47(1): 3-19. 
24. Davda J, Labhasetwar V (2002) Characterization of nanoparticle uptake by endothelial cells. Int J Pharm 233(1-2): 51-59.

25. Brigger I, Dubernet C, Couvreur P (2002) Nanoparticles in cancer therapy and diagnosis. Adv Drug Deliv Rev 54(5): 631-651.

26. Yan J, Wang Y, Zhang X, Liu S, Tian C, et al. (2016) Targeted nanomedicine for prostate cancer therapy: docetaxel and curcumin co-encapsulated lipid-polymer hybrid nanoparticles for the enhanced anti-tumor activity in vitro and in vivo. Drug Deliv 23(5): 1757-1762.

27. Yeh CY, Hsiao JK, Wang YP, Lan CH, Wu HC (2016) Peptide-conjugated nanoparticles for targeted imaging and therapy of prostate cancer. Biomaterials 99: 1-15.

28. Baltazar F, Pinheiro C, Morais-Santos F, Azevedo-Silva J, Queirós 0, et al. (2014) Monocarboxylate transporters as targets and mediators in cancer therapy response. Histol Histopathol 29(12): 1511-1524.

29. Marrache S, Dhar S (2015) The energy blocker inside the power house: mitochondria targeted delivery of 3-bromopyruvate. Chem Sci 6(3): 1832-1845.

30. Sun SB, Liu P, Shao FM, Miao QL (2015) Formulation and evaluation of PLGA nanoparticles loaded capecitabine for prostate cancer. Int J Clin Exp Med 8(10): 19670-19681.

31. Diwan M, Elamanchili P, Cao M, Samuel J (2004) Dose sparing of CpG oligodeoxynucleotide vaccine adjuvants by nanoparticle delivery. Curr Drug Deliv 1(4): 405-412.

32. McCall RL, Sirianni RW (2013) PLGA nanoparticles formed by single-or double-emulsion with vitamin E- TPGS video link. J Vis Exp 82: 51015.

33. Hofmann D, Messerschmidt C, Bannwarth MB, Landfester K, Mailänder V (2014) Drug delivery without nanoparticle uptake: delivery by a kissand-run mechanism on the cell membrane. Chem Commun 50(11): $1369-1371$

34. Jewell C, Lopez SB, Irvine D (2012) Engineering the lymph node microenvironment via intranodal controlled release vaccine depots. J Immunol 188(Suppl 1): 58

35. Danhier F, Ansorena E, Silva JM, Coco R, Le Breton A, et al. (2012) PLGAbased nanoparticles: An overview of biomedical applications. J Control Release 161(2): 505-522.

36. Dhar S, Daniel WL, Giljohann DA, Mirkin CA, Lippard SJ (2009) Polyvalent oligonucleotide gold nanoparticle conjugates as delivery vehicles for platinum (IV) warheads. J Am Chem Soc 131(41): 14652-14653.

37. Qu X, Yao C, Wang J, Li Z, Zhang Z (2012) Anti-CD30-targeted gold nanoparticles for photothermal therapy of L-428 Hodgkin's cell. Int J Nanomedicine 7: 6095-6103
38. Leones R, Fernandes M, Ferreira RA, Cesarino I, Lima JF, et al. (2014) Luminescent DNA- and agar-based membranes. J Nanosci Nanotechnol 14(9): 6685-6691.

39. Xi H, Cun D, Xiang R, Guan Y, Zhang Y, et al. (2013) Intra-articular drug delivery from an optimized topical patch containing teriflunomide and lornoxicam for rheumatoid arthritis treatment: Does the topical patch really enhance a local treatment? J Control Release 169(1-2): 73-81.

40. Shukla R, Bansal V, Chaudhary M, Basu A, Bhonde RR, et al. (2005) Biocompatibility of gold nanoparticles and their endocytotic fate inside the cellular compartment: A microscopic overview. Langmuir 21(23): 10644-10654.

41. Rojo J, Díaz V, de la Fuente JM, Segura I, Barrientos AG, et al. (2004) Gold glyconanoparticles as new tools in antiadhesive therapy. ChemBioChem 5(3): 291-297.

42. Lv H, Wu P, Wan W, Mu S (2014) Electrochemical durability of heattreated carbon nanospheres as catalyst supports for proton exchange membrane fuel cells. J Nanosci Nanotechnol 14(9): 7027-7031.

43. Pernodet N, Fang X, Sun Y, Bakhtina A, Ramakrishnan A, et al. (2006) Adverse effects of citrate/gold nanoparticles on human dermal fibroblasts. Small 2(6): 766-773.

44. Hamdy S, Molavi O, Ma Z, Haddadi A, Alshamsan A, et al. (2008) Codelivery of cancer-associated antigen and toll-like receptor 4 ligand in PLGA nanoparticles induces potent CD8+ T cell-mediated anti-tumor immunity. Vaccine 26(39): 5046-5057.

45. Sanna V, Pintus G, Roggio AM, Punzoni S, Posadino AM, et al. (2011) Targeted biocompatible nanoparticles for the delivery of (-)-epigallocatechin 3-gallate to prostate cancer cells. J Med Chem 54(5): 1321-1332.

46. Chan JM, Valencia PM, Zhang L, Langer R, Farokhzad OC (2010) Polymeric nanoparticles for drug delivery. Methods Mol Biol 624: 163-175.

47. Chen S, Zhao X, Chen J, Chen J, Kuznetsova L, et al. (2010) Mechanismbased tumor-targeting drug delivery system. Validation of efficient vitamin receptor-mediated endocytosis and drug release. Bioconjug Chem 21(5): 979-987.

48.Zempleni J (2005) Uptake, localization, and noncarboxylase roles of biotin. Annu Rev Nutr 25: 75-196.

49. Gagliardi M, Bertero, Bifone A (2017) Molecularly imprinted biodegradable nanoparticles. Sci Rep doi:10.1038/srep40046.

50. Wilhelm S, Tavares AJ, Dai Q Ohta S, Dvorak HF, et al. (2016) Analysis of nanoparticle delivery to tumours. Perspectives 1: 16014.
Creative Commons Attribution 4.0 International License

For possible submissions Click Here

\section{Submit Article}

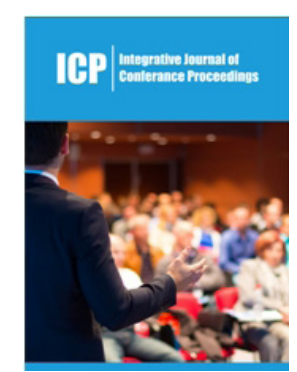

Integrative Journal of Conference Proceedings

\section{Benefits of Publishing with us}

- High-level peer review and editorial services

- Freely accessible online immediately upon publication

- Authors retain the copyright to their work

- Licensing it under a Creative Commons license

- Visibility through different online platforms 